\title{
Características clínicas de pacientes con pancreatitis aguda atendidos en un hospital de alta complejidad en Cali
}

\section{Clinical characteristics of patients with acute pancreatitis treated in a tertiary referral hospital in Cali}

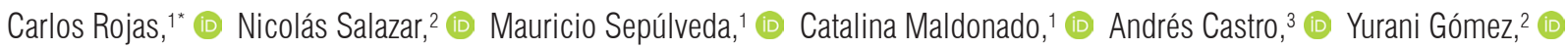
Edith Jurado, ${ }^{2}$ (D) Nelson Rojas. ${ }^{2}$ (D)

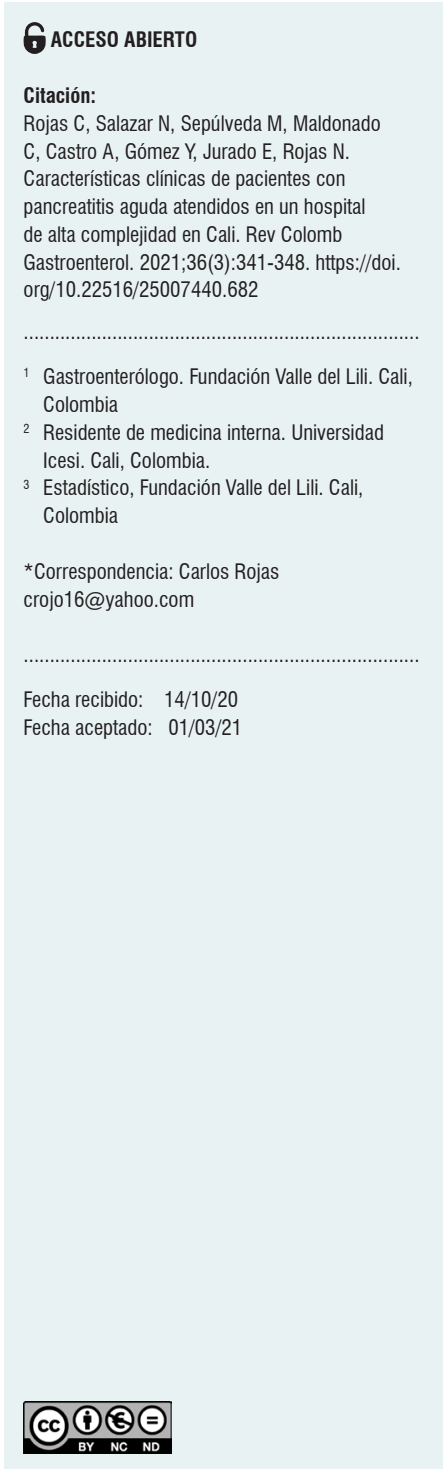

\begin{abstract}
Resumen
Introducción: la pancreatitis aguda (PA) es una enfermedad de alta complejidad clínica y, de acuerdo con su gravedad, puede tener una elevada morbimortalidad con altos costos para el sistema de salud, especialmente a nivel intrahospitalario. Materiales y métodos: se desarrolló un estudio descriptivo basado en historias clínicas de un hospital universitario de alta complejidad. Se revisaron las historias con diagnóstico CIE 10 de pancreatitis aguda entre enero de 2011 y diciembre de 2018. Se incluyeron todos los pacientes mayores de 18 años, de ambos sexos, con diagnóstico de PA por cumplimiento de al menos 2 de los criterios de Atlanta de 2012. Resultados: se revisaron 1353 historias clínicas, de las cuales 386 cumplieron criterios para PA. Entre ellas se identificaron 205 mujeres (53\%) y 181 hombres (47\%), y la prevalencia de comorbilidades fue inferior al $10 \%$. El $38 \%$ de los casos de pancreatitis ocurrieron en personas entre los 50 y 70 años de edad. Con respecto a la etiología de la PA, el origen biliar fue el de mayor frecuencia, con 200 casos del total (52 \%); seguido de idiopático (19,7\%) y poscolangiopancreatografía retrógrada endoscópica (CPRE), que ocurrió en 33 pacientes $(8,5 \%)$. Conclusiones: la PAes una entidad frecuente que afecta a adultos de todas las edades y genera una cantidad importante de consultas en urgencias. En Colombia, los datos previos apuntaban a pacientes con pancreatitis graves y no se tenía conocimiento del comportamiento sociodemográfico y clínico de las pancreatitis agudas en urgencias.
\end{abstract}

\section{Palabras clave}

Pancreatitis, aguda, características de la población.

\begin{abstract}
Introduction: Acute pancreatitis (AP) is a disease with a high degree of clinical complexity, and depending on its severity, it can have high morbidity and mortality rates, resulting in substantial health-care costs, particularly at the hospital level. Materials and methods: A descriptive study was developed based on the medical records of a tertiary referral university hospital. The records that included an ICD 10 diagnosis of acute pancreatitis between January 2011 and December 2018 were reviewed. All patients over the age of 18 , of both sexes, with an AP diagnosis who met at least two of the 2012 Atlanta criteria were included in the study. Results: 1353 records were reviewed, of which 386 met the criteria for AP. There were 205 women (53\%) and 181 males (47\%) among them, and comorbidities were found in less than $10 \%$ of the participants. $38 \%$ of cases of pancreatitis occurred in people between 50 and 70 years of age. Regarding the etiology of AP, biliary origin was the most frequent with 200 cases $(52 \%)$, followed by idiopathic $(19.7 \%)$ and post-endoscopic retrograde cholangiopancreatography (ERCP) in 33 patients (8.5\%). Conclusions: AP is a common condition that affects adults of all ages and results in a high number of emergency room visits. Previous data in Colombia was only available for individuals with severe pancreatitis, and nothing was known about the sociodemographic and clinical characteristics of acute pancreatitis in the emergency room.
\end{abstract}

\section{Keywords}

Pancreatitis; Acute; Characteristics of the population. 


\section{INTRODUCCIÓN}

La pancreatitis aguda (PA) es un proceso inflamatorio que compromete el páncreas, secundaria a la autodigestión por parte de las enzimas que este secreta ${ }^{(1)}$. Se debe sospechar en un paciente con dolor abdominal superior, agudo y grave; y requiere para su diagnóstico evidencia bioquímica o radiológica ${ }^{(1-4)}$.

Es una enfermedad de alta complejidad clínica y, de acuerdo a su gravedad, puede tener elevada morbimortalidad ${ }^{(5)}$ con altos costos para el sistema de salud, especialmente a nivel intrahospitalario ${ }^{(6,7)}$.

En Colombia se cuenta con pocos estudios que caractericen a los pacientes con PA. El presente trabajo buscó describir las características sociodemográficas y clínicas de los pacientes con diagnóstico de PA en una institución de cuarto nivel entre los años 2011 y 2018.

\section{MATERIALES Y MÉTODOS}

Se desarrolló un estudio descriptivo retrospectivo, basado en historias clínicas de un hospital universitario de alta complejidad. Luego de obtener la aprobación del comité de ética institucional, se revisaron las historias con diagnóstico CIE 10 de pancreatitis aguda entre enero de 2011 y diciembre de 2018 (CIE 10 K85X, K868, K871). Se incluyeron todos los pacientes mayores de 18 años, de ambos sexos, con diagnóstico de PA por cumplimiento de al menos 2 de los criterios de Atlanta de $2012^{(1)}$. Se describieron las características sociodemográficas, presentación clínica, niveles de enzimas pancreáticas, imágenes diagnosticas, manejo y complicaciones.

Se realizó un análisis estadístico descriptivo; las variables continuas se expresan como mediana y rango intercuartílico (RIC), debido a su distribución. Las variables categóricas se presentan en proporciones. Para el análisis de datos se utilizó el software estadístico Stata 14.0.

\section{RESULTADOS}

Se revisaron 1353 historias clínicas, de las cuales 386 cumplieron criterios para PA. Entre ellas se identificaron 205 mujeres (53\%) y 181 hombres (47\%), y la prevalencia de comorbilidades fue inferior al $10 \%$. El $38 \%$ de los casos de pancreatitis ocurrieron en personas entre los 50 y 70 años de edad. En la Tabla 1 se pueden observar las proporciones de los demás grupos etarios.

En cuanto a la manifestación clínica inicial, se encontró que el 97,4\% de los pacientes se presentó con dolor abdominal y $73 \%$ con emesis, que son los dos síntomas más frecuentes; la frecuencia de otros síntomas se describe en la Tabla 1. En 98 pacientes $(25,7 \%)$ que consultaron a urgen-
Tabla 1. Datos sociodemográficos y presentación clínica

\begin{tabular}{|c|c|c|}
\hline Variable & n (total: 386) & $\%$ \\
\hline \multicolumn{3}{|l|}{ Sexo } \\
\hline - Masculino & 181 & 46,9 \\
\hline - Femenino & 205 & 53,1 \\
\hline \multicolumn{3}{|l|}{ Edad (años) } \\
\hline$-<30$ & 55 & 14,3 \\
\hline$-30-49$ & 107 & 27,7 \\
\hline$-50-69$ & 148 & 38,3 \\
\hline$->70$ & 76 & 19,7 \\
\hline \multicolumn{3}{|l|}{ Antecedentes } \\
\hline - Dislipidemia & 30 & 7,8 \\
\hline - Alcoholismo & 21 & 5,4 \\
\hline - Tabaquismo & 21 & 5,4 \\
\hline - Pancreatitis recurrente & 13 & 3,4 \\
\hline \multicolumn{3}{|l|}{ Presentación clínica } \\
\hline - Dolor abdominal & 376 & 97,4 \\
\hline - Emesis & 282 & 73,1 \\
\hline - SIRS* & 138 & 35,8 \\
\hline - Pancreatitis aguda severa & 98 & 25,7 \\
\hline - Ictericia & 75 & 19,4 \\
\hline - Choque & 58 & 15,0 \\
\hline - Tiempo de síntomas ${ }^{* *}$ (horas) & $20(4-48)$ & - \\
\hline
\end{tabular}

*SIRS: síndrome de respuesta inflamatoria sistémica. Se define como la presencia de más de dos de los siguientes: temperatura $>38^{\circ} \mathrm{C}$ o $<36$ ${ }^{\circ} \mathrm{C}$, frecuencia cardíaca (FC) $>90$ latidos por minuto (lpm), frecuencia respiratoria $(\mathrm{FR})>20$ respiraciones por minuto $(\mathrm{rpm})$, leucocitos $>$ $10000,<4000$ o bandemia superior al $10 \%$. ${ }^{* *}$ Mediana (RIC).

cias se diagnosticó PA grave. Las enzimas pancreáticas se encontraron elevadas en la mayoría de los casos: la amilasa y la lipasa se midieron en $36 \%$ y $84 \%$, respectivamente. La mediana de amilasa fue 874 (valor límite de laboratorio = 100) y 1293 para la lipasa (valor límite de laboratorio $=60$ ). El tiempo de duración de los síntomas previa al ingreso a urgencias fue en promedio de 20 horas (8-48 horas).

Con respecto a la etiología de la PA, el origen biliar fue el de mayor frecuencia, con 200 casos del total (52\%); seguido de idiopático $(19,7 \%)$; poscolangiopancreatografía retrógrada endoscópica, (CPRE) que ocurrió en 33 pacientes $(8,5 \%)$; 
y tóxico en 22 casos (5,7 \%) (Tabla 2). Otras causas menos frecuentes se mencionan en la (Tabla 3 ).

Tabla 2. Tóxicos

\begin{tabular}{lll}
\hline \multicolumn{1}{r}{ Medicamento } & $\mathbf{n}$ & $\%$ \\
\hline Naturista & 5 & 22,8 \\
\hline Azatioprina & 5 & 22,8 \\
\hline Pembrolizumab & 1 & 4,5 \\
\hline 5-ASA & 1 & 4,5 \\
\hline Clozapina & 1 & 4,5 \\
\hline Docetaxel & 1 & 4,5 \\
\hline Glucantime & 1 & 4,5 \\
\hline Sulfonamidas & 1 & 4,5 \\
\hline Aciclovir & 1 & 4,5 \\
\hline AlNE & 1 & 4,5 \\
\hline Antirretrovirales & 1 & 4,5 \\
\hline Corticoides & 1 & 4,5 \\
\hline Estrógenos & 1 & 4,5 \\
\hline Ácido valproico & 1 & 4,5 \\
\hline
\end{tabular}

5-ASA: ácido 5 aminosalicílico; AINE: antiinflamatorios no esteroideos.

Tabla 3. Etiología

\begin{tabular}{lcc|}
\hline \multicolumn{1}{c}{ Causa } & n (total: $\mathbf{3 8 6}$ ) & \% \\
\hline Biliar & 200 & 51,8 \\
\hline Idiopática & 76 & 19,7 \\
\hline Pos-CPRE & 33 & 8,5 \\
\hline Tóxica & 22 & 5,7 \\
\hline Alcohólica & 13 & 3,4 \\
\hline Postraumática & 12 & 3,1 \\
\hline Recurrente & 10 & 2,6 \\
\hline Dislipidémica & 9 & 2,3 \\
\hline Posquirúrgica & 6 & 1,6 \\
\hline Autoinmune & 5 & 1,3
\end{tabular}

Como parte del diagnóstico se realizó ecografía biliar a todos los pacientes, 131 fueron llevados posteriormente a tomografía axial computarizada (TAC) abdominal $(30,1 \%)$ y 137 a colangiorresonancia $(35,5 \%)$. El hallazgo de pancreatitis en dichos estudios fue del $22,8 \%, 80,2 \%$ y $64,8 \%$, respectivamente. Se usó la clasificación de Balthazar en todos los pacientes con TAC y se clasificó a 47 pacientes como A (35,9\%), 27 pacientes como B $(20,6 \%), 28$ pacientes como C $(21,4 \%), 18$ pacientes como D (13,7\%) y 11 pacientes como E (8,4 \%). De los pacientes evaluados, 42 (10\%) presentaron necrosis pancreática, de los cuales $23(54,8 \%)$ tenían necrosis en menos del $30 \%$ del páncreas, 11 (26,2\%) entre el $30 \%$ y $50 \%$ y 8 (19\%) en más del $50 \%$.

En cuanto al manejo, 372 pacientes fueron hospitalizados en el piso general $(96,4 \%)$ con una mediana de estancia hospitalaria de 4 (2-7) días. 230 pacientes requirieron manejo en la unidad de cuidados intensivos (UCI; 59,6\%) con una mediana de estancia en la UCI de 4 (2-9) días. En relación con el inicio de la vía oral, fue en promedio 2 (1-3) días después del ingreso en el 81,5\% de los pacientes, nutrición enteral en 10,6 \% y nutrición parenteral en 4,1\% de los pacientes. Otras intervenciones como tratamiento antibiótico y procedimientos se describen en la Tabla 4.

Tabla 4. Manejo

\begin{tabular}{lcc|}
\multicolumn{1}{c}{ Manejo } & n (total: 386 ) & $\%$ \\
\hline Hospitalización & & \\
- Piso & 372 & $96,4 \%$ \\
- Días en piso & $4^{*}$ & $2-^{* *}$ \\
\hline - UCl & 230 & $59,6 \%$ \\
\hline - Días en la UCl & $4^{*}$ & $2-9^{* *}$ \\
\hline Alimentación & & \\
\hline - Tiempo sin vía oral & $2^{*}$ & $1-3^{* *}$ \\
\hline - Oral & 315 & $81,6 \%$ \\
\hline - Enteral & 41 & $10,6 \%$ \\
\hline - Parenteral & 16 & $4,1 \%$ \\
\hline Antibiótico & & \\
\hline - Requerimiento antibiótico & 104 & $26,9 \%$ \\
\hline Indicación & & \\
\hline - Pancreática & 27 & $26 \%$ \\
\hline - Extra pancreática & 77 & $74 \%$ \\
\hline Procedimientos & & \\
\hline - CPRE & 13 & $3,4 \%$ \\
\hline - Colecistectomía laparoscópica & 126 & $32,6 \%$ \\
\hline - Colecistectomía, laparotomía & $13 \%$ \\
\hline - Necrosectomía & 19 & 59 \\
\hline
\end{tabular}

*Mediana. **RIC. 
Un total de 323 pacientes $(83,6 \%)$ no presentaron complicaciones, $42(10,9 \%)$ presentaron pancreatitis necrotizante; 9, pseudoquiste pancreático (2,3\%); 8 (2,1\%), absceso pancreático, y 4 (1,0\%), diabetes mellitus (DM). La mortalidad general fue del 4,1 \% (17 pacientes) (Tabla 5).

Tabla 5. Complicaciones

\begin{tabular}{|lcc|}
\hline \multicolumn{1}{c}{ Complicaciones } & n (total: $\mathbf{3 8 6})$ & \% \\
\hline Sin complicaciones & 323 & 83,6 \\
\hline Pancreatitis necrotizante & 42 & 10,9 \\
\hline Pseudoquiste pancreático & 9 & 2,3 \\
\hline Absceso pancreático & 8 & 2,1 \\
\hline DM & 4 & 1,0 \\
\hline Muerte & 17 & 4,4 \\
\hline
\end{tabular}

\section{DISCUSIÓN}

En este estudio se incluyeron 386 pacientes que cumplieron con los criterios para el diagnóstico de PA durante un período de 8 años, luego de una revisión de más de 1300 historias clínicas que tenían un diagnóstico CIE-10 de PA. Esto supone que, aún con cuadros clínicos sugestivos de esta patología, son limitados los casos que sí cumplen con los criterios. La proporción de pacientes por distribución por sexos fue similar a la de estudios internacionales ${ }^{(8-10)} \mathrm{y}$ locales $^{(11,12)}$, con un ligero predominio del sexo femenino, situación que puede estar relacionada con la etiología biliar como causa más frecuente de PA, que suele ser ligeramente más frecuente en mujeres ${ }^{(8,9)}$. La prevalencia fue mayor en pacientes entre 50 y 70 años, lo que es consistente con el estudio de Yatay y colaboradores, en el que la edad más prevalente fue la sexta década de la vida ${ }^{(9)}$. Se presenta un bajo porcentaje de comorbilidades que se puede explicar por las variables elegidas para el estudio, que son aquellas que se asocian como eventos precipitantes de PA, y se puede observar que solo una pequeña fracción de los pacientes con estos factores predisponentes desarrolla la enfermedad.

El dolor abdominal característico de predominio epigástrico y con irradiación en banda fue el síntoma más frecuente, presente encasilatotalidaddelospacientes (97,4\%), porcentaje similar a lo reportado en la literatura, en la que se reporta que este es el síntoma cardinal que lleva a los pacientes a consultar al servicio de urgencias ${ }^{(1)}$. Cerca de tres de cada cuatro pacientes presentaron episodios eméticos y en uno de cada cinco de los que consultó se documentó ictericia; esta frecuencia de síntomas es parecida a lo reportado ${ }^{(1)}$.
En relación con las enzimas pancreáticas, por una parte, la amilasa sérica presenta una elevación más temprana y ayuda a orientar el diagnóstico para PA, pero presenta igualmente un rápido regreso a la normalidad, por lo que se puede perder el diagnóstico de PA en pacientes que se presentan más de 24 horas después del inicio de los síntomas. Por otra parte, la lipasa sérica, a pesar de presentar una elevación más tardía, permanece elevada por más tiempo, pues alcanza su punto máximo a las 24 horas y perdura hasta 14 días $^{(3)}$. Asociado a esto, en nuestro estudio vemos que en promedio los pacientes ingresan a urgencias a las 20 horas del inicio de los síntomas y se encuentran casos de hasta 48 horas posteriores, esto podría indicar el mayor porcentaje de lipasa sérica realizada ( $84 \%)$, en comparación con amilasa sérica (36\%). Las dos tienen una buena especificidad y sensibilidad ${ }^{(1)}$. Los pacientes que se incluyeron en el estudio cumplían criterios de elevación de enzimas pancreáticas. Además, en el protocolo institucional se pueden usar amilasa o lipasa, generalmente según las guías institucionales se toman en las primeras 24-48 horas.

En cuanto a la etiología de PA, el origen biliar sigue siendo la principal causa con más de la mitad de los casos reportados, hallazgo que es consistente con múltiples revisiones previas alrededor del mundo ${ }^{(8,13)}$, y ligeramente inferior a lo reportado por Díaz y colaboradores en $2012^{(11)}$; sin embargo, en contraste con otros estudios previos, en nuestra población la segunda causa más frecuente no fue la alcohólica, que solo representó el 3,4 \% de los casos; considerablemente menos que en estudios internacionales ${ }^{(8,13)}$ en los que representa alrededor del $20 \%$. Nuestros hallazgos son consistentes con lo reportado por Díaz y colaboradores ${ }^{(11)}$, situación que hace considerar la posibilidad de un menor consumo de alcohol a nivel nacional o mutaciones locales de la aldehídodeshidrogenasa (ALDH), alcohol-deshidrogenasa $(\mathrm{ADH})$ y citocromo P450 CYP2E1, que hacen que las personas sean menos propensas a desarrollar la enfermedad; consideración que se tendrá que estudiar en el futuro ${ }^{(8,14-19)}$.

La tercera causa de PA en este estudio fue pos-CPRE, la definición que se usó fue elevación de amilasas 3 veces al valor normal, 24 horas después del procedimiento, esta etiología representó cerca del $8 \%$ de los casos; una incidencia muy elevada cuando se compara con los estudios en otros países ${ }^{(8,13,20,21)}$ y casi el doble de lo reportado por Díaz $y$ colaboradores ${ }^{(11)}$, hallazgo que puede estar relacionado con la complejidad de los casos atendidos en la institución, un centro de remisión del suroccidente colombiano ${ }^{(22)}$. En concordancia con esto, se encontró que la PA grave posterior a CPRE fue poco frecuente en comparación con las otras etiologías (3\%); sin embargo, en nuestro estudio esta incidencia es elevada en comparación con lo reportado en la literatura $(0,5 \%)^{(8)}$. 
La hipertrigliceridemia fue una causa infrecuente de PA en nuestro estudio, representando solo $2,3 \%$ de los casos con un promedio de triglicéridos de $600 \mathrm{mg} / \mathrm{dL}$, sustancialmente menor a lo reportado por estudios como el de Papachristou y colaboradores ${ }^{(23)}$, en el que llega a representar hasta un $10 \%$ de los casos, y casi la mitad de lo reportado por Díaz y colaboradores ${ }^{(11)}$ a nivel local. Aunque en más del $80 \%$ de los casos se logró distinguir una etiología plausible de PA, en algunos pacientes (especialmente en aquellos en los que se determinó causa idiopática) no se dispuso de un perfil lipídico.

Otras causas de pancreatitisfueronla toxicidad, con un $2 \%$, valor menor al reportado en otras series $(5,7 \%)^{(8,24,25)}$ y el trauma, en el $3 \%$ de los casos, inferior a lo descrito previamente a nivel nacional ${ }^{(11)}$.

En cuanto a la relación entre el valor de enzimas pancreáticas y la gravedad de la enfermedad, no se logró establecer una relación compatible con los hallazgos de revisiones encontradas en la literatura. En los 73 pacientes que presentaron lipasas inferiores a $600 \mathrm{UI} / \mathrm{mL}$, la mortalidad fue del 5,7\%; mientras que, en aquellos con valores superiores, la mortalidad fue del 3,3\% ${ }^{(26)}$.

Casi el $60 \%$ de los pacientes requirió manejo en la UCI en las primeras 48 horas, el doble de lo reportado en otras series $^{(8,11)}$, posiblemente relacionado con las características de población típica de un centro de remisión, como se mencionó anteriormente, donde tiende a ser frecuente un nivel de complejidad mayor. Se definió PA grave según los criterios de Atlanta, el criterio médico y en el APACHE II.

El 26,9 \% de los pacientes recibió antibióticos; de estos, el $74 \%$ fue debido a infecciones extrapancreáticas ${ }^{(27-30)}$; no se usó profilaxis antibiótica, lo que concuerda con el manejo planteado en las guías ${ }^{(11)}$.

El $30 \%$ de los pacientes fue llevado a colecistectomía, teniendo en cuenta que la etiología más frecuente fue de origen biliar y el procedimiento se realizó durante la misma hospitalización. Mientras que solo el $5 \%$ de los pacientes fue llevado a necrosectomía. Comparado con otras series nacionales, se encontró una necesidad menor de procedimientos, lo que podría estar relacionado con una inclusión de menor cantidad de casos graves y un manejo oportuno ${ }^{(31-37)}$.
A diferencia de otras series, el 83,6\% de los pacientes no presentó complicaciones. La complicación más frecuente fue la necrosis pancreática $(10,9 \%)$, seguida del pseudoquiste pancreático $(2,3 \%)$ y el absceso pancreático $(2,1$ $\%)$; más allá de todo ello, la mortalidad evidenciada en el estudio fue menor a la reportada en otras series con un valor del $4,4 \%^{(38-40)}$ y la mortalidad referida en la historia clínica se relacionó con la pancreatitis. Se revisaron las 17 historias clínicas de los pacientes fallecidos, en las cuales se analizó la causa de muerte y el $95 \%$ falleció por complicaciones infecciosas. Cuando se revisa la relación entre complicaciones y gravedad de la pancreatitis, las pancreatitis leves solo se complicaron en el $3 \%$ de los casos, mientras que las graves tuvieron tasas de complicación hasta del $37 \%{ }^{(41-45)}$. El alcance de este estudio no consideraba la descripción del manejo de las complicaciones, que los tendremos en cuenta para un estudio adicional, dado que en nuestra institución realizamos necrosectomías transgástricas y drenajes con prótesis de pseudoquistes transgástricos.

\section{CONCLUSIONES}

La PA es una entidad frecuente que afecta a adultos de todas las edades y genera una cantidad importante de consultas en urgencias. En Colombia, los datos previos apuntaban a pacientes con pancreatitis graves y no se tenía conocimiento del comportamiento clínico de las pancreatitis agudas en urgencias. Este estudio aporta información clínica de interés sobre una población extensa de pacientes tratados en un centro de remisión. El comportamiento demográfico de la población estudiada es similar a lo reportado en el resto del mundo, con afección parecida en sexos y grupos etarios. Es de resaltar que la causa biliar es la principal etiología de la PA y, en la mayoría de las ocasiones, su estudio extenso permitió esclarecer otras etiologías. El manejo en general concuerda con la mayoría de guías en las que se sugiere evitar el uso de antibióticos profilácticos y diferir la alimentación temprana. Se requieren de más estudios locales para esclarecer el comportamiento de las complicaciones y mortalidad a largo plazo en nuestro medio.

\section{REFERENCIAS}

1. Banks PA, Bollen TL, Dervenis C, Gooszen HG, Johnson CD, Sarr MG, Tsiotos GG, Vege SS; Acute Pancreatitis Classification Working Group. Classification of acute pancreatitis--2012: revision of the Atlanta classification and definitions by international consensus. Gut.
2013;62(1):102-11

https://doi.org/10.1136/gutjnl-2012-302779

2. Working Party of the British Society of Gastroenterology; Association of Surgeons of Great Britain and Ireland; Pancreatic Society of Great Britain and Ireland; Association of Upper GI Surgeons of Great Britain and Ireland. UK 
guidelines for the management of acute pancreatitis. Gut. 2005;54 Suppl 3(Suppl 3):iiil-9.

https://doi.org/10.1136/gut.2004.057026

3. Tenner S, Baillie J, DeWitt J, Vege SS; American College of Gastroenterology. American College of Gastroenterology guideline: management of acute pancreatitis. Am J Gastroenterol. 2013;108(9):1400-15; 1416. https://doi.org/10.1038/ajg.2013.218

4. Arvanitakis M, Delhaye M, De Maertelaere V, Bali M, Winant C, Coppens E, Jeanmart J, Zalcman M, Van Gansbeke D, Devière J, Matos C. Computed tomography and magnetic resonance imaging in the assessment of acute pancreatitis. Gastroenterology. 2004;126(3):715-23. https://doi.org/10.1053/j.gastro.2003.12.006

5. Di MY, Liu H, Yang ZY, Bonis PA, Tang JL, Lau J. Prediction Models of Mortality in Acute Pancreatitis in Adults: A Systematic Review. Ann Intern Med. 2016;165(7):482-490. https://doi.org/10.7326/M16-0650

6. Pandol SJ, Saluja AK, Imrie CW, Banks PA. Acute pancreatitis: bench to the bedside. Gastroenterology. 2007; 132(3):1127-51.

https://doi.org/10.1053/j.gastro.2007.01.055

7. Fagenholz PJ, Castillo CF, Harris NS, Pelletier AJ, Camargo CA Jr. Increasing United States hospital admissions for acute pancreatitis, 1988-2003. Ann Epidemiol. 2007; 17(7):491-7. https://doi.org/10.1016/j.annepidem.2007.02.002

8. Weiss FU, Laemmerhirt F, Lerch MM. Etiology and Risk Factors of Acute and Chronic Pancreatitis. Visc Med. 2019;35(2):73-81. https://doi.org/10.1159/000499138

9. Yadav D, Lowenfels AB. Trends in the epidemiology of the first attack of acute pancreatitis: a systematic review. Pancreas. 2006;33(4):323-30. https://doi.org/10.1097/01.mpa.0000236733.31617.52

10. Yadav D, Lowenfels AB. The epidemiology of pancreatitis and pancreatic cancer. Gastroenterology. 2013;144(6):1252-61. https://doi.org/10.1053/j.gastro.2013.01.068

11. Diaz C, Garzón S, Morales CH, Montoya M. Pancreatitis aguda grave: curso clínico, manejo y factores asociados con mortalidad. Rev Colomb Cir. 2012;27(4):281-9.

12. Puerto LH, Medina R, Nuñez LR, Conrado Jimenez H, Olaya JG, San Juan JF, Puerto Horta SA, Martínez Montalvo CM, Medina Cabrera MM. Manejo y desenlaces de la pancreatitis aguda en un hospital de cuarto nivel (Huila, Colombia), 3 años de experiencia. Rev Colomb Gastroenterol. 2019; 34 (1): 11-16. https://doi.org/10.22516/25007440.243

13. Bustamante Durón D, García Laínez A, Umanzor García W, Leiva Rubio L, Barrientos Rosales A, Diek Romero L. Pancreatitis aguda: evidencia actual. Archivos de Medicina. 2018;14(1:4):1-10.

14. Bertin C, Pelletier AL, Vullierme MP, Bienvenu T, Rebours V, Hentic O, Maire F, Hammel P, Vilgrain V, Ruszniewski
P, Lévy P. Pancreas divisum is not a cause of pancreatitis by itself but acts as a partner of genetic mutations. Am J Gastroenterol. 2012;107(2):311-7. https://doi.org/10.1038/ajg.2011.424

15. DiMagno MJ, Dimagno EP. Pancreas divisum does not cause pancreatitis, but associates with CFTR mutations. Am J Gastroenterol. 2012;107(2):318-20. https://doi.org/10.1038/ajg.2011.430

16. Whitcomb DC, LaRusch J, Krasinskas AM, Klei L, Smith JP, Brand RE, Neoptolemos JP, Lerch MM, Tector M, Sandhu BS, Guda NM, Orlichenko L; Alzheimer's Disease Genetics Consortium, Alkaade S, Amann ST, Anderson MA, Baillie J, Banks PA, Conwell D, Coté GA, Cotton PB, DiSario J, Farrer LA, Forsmark CE, Johnstone M, Gardner TB, Gelrud A, Greenhalf W, Haines JL, Hartman DJ, Hawes RA, Lawrence C, Lewis M, Mayerle J, Mayeux R, Melhem NM, Money ME, Muniraj T, Papachristou GI, Pericak-Vance MA, Romagnuolo J, Schellenberg GD, Sherman S, Simon P, Singh VP, Slivka A, Stolz D, Sutton R, Weiss FU, Wilcox CM, Zarnescu NO, Wisniewski SR, O’Connell MR, Kienholz ML, Roeder K, Barmada MM, Yadav D, Devlin B. Common genetic variants in the CLDN2 and PRSS1-PRSS2 loci alter risk for alcohol-related and sporadic pancreatitis. Nat Genet. 2012;44(12):1349-54. https://doi.org/10.1038/ng.2466

17. Apte MV, Pirola RC, Wilson JS. Mechanisms of alcoholic pancreatitis. J Gastroenterol Hepatol. 2010;25(12):1816-26. https://doi.org/10.1111/j.1440-1746.2010.06445.x

18. Phillip V, Huber W, Hagemes F, Lorenz S, Matheis U, Preinfalk S, Schuster T, Lippl F, Saugel B, Schmid RM. Incidence of acute pancreatitis does not increase during Oktoberfest, but is higher than previously described in Germany. Clin Gastroenterol Hepatol. 2011;9(11):995-1000.e3. https://doi.org/10.1016/j.cgh.2011.06.016

19. Whitcomb DC. Genetic risk factors for pancreatic disorders. Gastroenterology. 2013;144(6):1292-302. https://doi.org/10.1053/j.gastro.2013.01.069

20. Dumonceau JM, Andriulli A, Deviere J, Mariani A, Rigaux J, Baron TH, Testoni PA; European Society of Gastrointestinal Endoscopy. European Society of Gastrointestinal Endoscopy (ESGE) Guideline: prophylaxis of post-ERCP pancreatitis. Endoscopy. 2010;42(6):503-15. https://doi.org/10.1055/s-0029-1244208

21. Aktas H, Mensink PB, Haringsma J, Kuipers EJ. Low incidence of hyperamylasemia after proximal double-balloon enteroscopy: has the insertion technique improved? Endoscopy. 2009;41(8):670-3. https://doi.org/10.1055/s-0029-1214976

22. Ding X, Zhang F, Wang Y. Risk factors for post-ERCP pancreatitis: A systematic review and meta-analysis. Surgeon. 2015;13(4):218-29. https://doi.org/10.1016/j.surge.2014.11.005

23. Papachristou GI, Machicado JD, Stevens T, Goenka MK, Ferreira M, Gutierrez SC, Singh VK, Kamal A, Gonzalez- 
Gonzalez JA, Pelaez-Luna M, Gulla A, Zarnescu NO, Triantafyllou K, Barbu ST, Easler J, Ocampo C, Capurso G, Archibugi L, Cote GA, Lambiase L, Kochhar R, Chua T, Tiwari SC, Nawaz H, Park WG, de-Madaria E, Lee PJ, Wu BU, Greer PJ, Dugum M, Koutroumpakis E, Akshintala V, Gougol A. Acute pancreatitis patient registry to examine novel therapies in clinical experience (APPRENTICE): an international, multicenter consortium for the study of acute pancreatitis. Ann Gastroenterol. 2017;30(1):106-113. https://doi.org/10.20524/aog.2016.0109

24. Nitsche C, Maertin S, Scheiber J, Ritter CA, Lerch MM, Mayerle J. Drug-induced pancreatitis. Curr Gastroenterol Rep. 2012;14(2):131-8. https://doi.org/10.1007/s11894-012-0245-9

25. Bertilsson S, Kalaitzakis E. Acute Pancreatitis and Use of Pancreatitis-Associated Drugs: A 10-Year Population-Based Cohort Study. Pancreas. 2015;44(7):1096-104. https://doi.org/10.1097/MPA.0000000000000406

26. Hamer JF. Retrospective cohort study demonstrates that modified CT Severity Index directly correlates with lipase values at or above 600. Ann Med Surg (Lond). 2020;56:169-172.

https://doi.org/10.1016/j.amsu.2020.06.023

27. Isenmann R, Rünzi M, Kron M, Kahl S, Kraus D, Jung N, Maier L, Malfertheiner P, Goebell H, Beger HG; German Antibiotics in Severe Acute Pancreatitis Study Group. Prophylactic antibiotic treatment in patients with predicted severe acute pancreatitis: a placebo-controlled, doubleblind trial. Gastroenterology. 2004;126(4):997-1004. https://doi.org/10.1053/j.gastro.2003.12.050

28. Dellinger EP, Tellado JM, Soto NE, Ashley SW, Barie PS, Dugernier T, Imrie CW, Johnson CD, Knaebel HP, Laterre PF, Maravi-Poma E, Kissler JJ, Sanchez-Garcia M, Utzolino S. Early antibiotic treatment for severe acute necrotizing pancreatitis: a randomized, double-blind, placebo-controlled study. Ann Surg. 2007;245(5):674-83. https://doi.org/10.1097/01.sla.0000250414.09255.84

29. Lim CL, Lee W, Liew YX, Tang SS, Chlebicki MP, Kwa AL. Role of antibiotic prophylaxis in necrotizing pancreatitis: a meta-analysis. J Gastrointest Surg. 2015;19(3):480-91. https://doi.org/10.1007/s11605-014-2662-6

30. Villatoro E, Mulla M, Larvin M. Antibiotic therapy for prophylaxis against infection of pancreatic necrosis in acute pancreatitis. Cochrane Database Syst Rev. 2010;2010(5):CD002941. https://doi.org/10.1002/14651858.CD002941.pub3

31. Gurusamy KS, Nagendran M, Davidson BR. Early versus delayed laparoscopic cholecystectomy for acute gallstone pancreatitis. Cochrane Database Syst Rev. 2013;(9):CD010326. https://doi.org/10.1002/14651858.CD010326.pub2

32. van Baal MC, Besselink MG, Bakker OJ, van Santvoort HC, Schaapherder AF, Nieuwenhuijs VB, Gooszen HG, van Ramshorst B, Boerma D; Dutch Pancreatitis Study Group. Timing of cholecystectomy after mild biliary pancreatitis: a systematic review. Ann Surg. 2012;255(5):860-6. https://doi.org/10.1097/SLA.0b013e3182507646

33. da Costa DW, Bouwense SA, Schepers NJ, Besselink MG, van Santvoort HC, van Brunschot S, Bakker OJ, Bollen TL, Dejong $\mathrm{CH}$, van Goor $\mathrm{H}$, Boermeester MA, Bruno MJ, van Eijck CH, Timmer R, Weusten BL, Consten EC, Brink MA, Spanier BWM, Bilgen EJS, Nieuwenhuijs VB, Hofker HS, Rosman C, Voorburg AM, Bosscha K, van Duijvendijk P, Gerritsen JJ, Heisterkamp J, de Hingh IH, Witteman BJ, Kruyt PM, Scheepers JJ, Molenaar IQ, Schaapherder AF, Manusama ER, van der Waaij LA, van Unen J, Dijkgraaf MG, van Ramshorst B, Gooszen HG, Boerma D; Dutch Pancreatitis Study Group. Same-admission versus interval cholecystectomy for mild gallstone pancreatitis (PONCHO): a multicentre randomised controlled trial. Lancet. 2015;386(10000):1261-1268. https://doi.org/10.1016/S0140-6736(15)00274-3

34. Freeman ML, Werner J, van Santvoort HC, Baron TH, Besselink MG, Windsor JA, Horvath KD, vanSonnenberg E, Bollen TL, Vege SS; International Multidisciplinary Panel of Speakers and Moderators. Interventions for necrotizing pancreatitis: summary of a multidisciplinary consensus conference. Pancreas. 2012;41(8):1176-94. https://doi.org/10.1097/MPA.0b013e318269c660

35. van Santvoort HC, Besselink MG, Bakker OJ, Hofker HS, Boermeester MA, Dejong CH, van Goor $\mathrm{H}$, Schaapherder AF, van Eijck CH, Bollen TL, van Ramshorst B, Nieuwenhuijs VB, Timmer R, Laméris JS, Kruyt PM, Manusama ER, van der Harst E, van der Schelling GP, Karsten T, Hesselink EJ, van Laarhoven CJ, Rosman C, Bosscha K, de Wit RJ, Houdijk AP, van Leeuwen MS, Buskens E, Gooszen HG; Dutch Pancreatitis Study Group. A step-up approach or open necrosectomy for necrotizing pancreatitis. N Engl J Med. 2010;362(16):1491-502. https://doi.org/10.1056/NEJMoa0908821

36. Bakker OJ, van Santvoort HC, van Brunschot S, Geskus RB, Besselink MG, Bollen TL, van Eijck CH, Fockens P, Hazebroek EJ, Nijmeijer RM, Poley JW, van Ramshorst B, Vleggaar FP, Boermeester MA, Gooszen HG, Weusten BL, Timmer R; Dutch Pancreatitis Study Group. Endoscopic transgastric vs surgical necrosectomy for infected necrotizing pancreatitis: a randomized trial. JAMA. 2012;307(10):1053-61. https://doi.org/10.1001/jama.2012.276

37. Mouli VP, Sreenivas V, Garg PK. Efficacy of conservative treatment, without necrosectomy, for infected pancreatic necrosis: a systematic review and meta-analysis. Gastroenterology. 2013;144(2):333-340.e2. https://doi.org/10.1053/j.gastro.2012.10.004

38. Wu D, Xu Y, Zeng Y, Wang X. Endocrine pancreatic function changes after acute pancreatitis. Pancreas. 2011;40(7):1006-11. https://doi.org/10.1097/MPA.0b013e31821fde3f

39. Pezzilli R, Simoni P, Casadei R, Morselli-Labate AM. Exocrine pancreatic function during the early recovery 
phase of acute pancreatitis. Hepatobiliary Pancreat Dis Int. 2009;8(3):316-9.

40. Symersky T, van Hoorn B, Masclee AA. The outcome of a long-term follow-up of pancreatic function after recovery from acute pancreatitis. JOP. 2006;7(5):447-53.

41. Doepel M, Eriksson J, Halme L, Kumpulainen T, Höckerstedt K. Good long-term results in patients surviving severe acute pancreatitis. Br J Surg. 1993;80(12):1583-6. https://doi.org/10.1002/bjs.1800801229

42. Lankisch PG, Breuer N, Bruns A, Weber-Dany B, Lowenfels AB, Maisonneuve P. Natural history of acute pancreatitis: a long-term population-based study. Am J Gastroenterol. 2009; 104(11):2797-805; quiz 2806. https://doi.org/10.1038/ajg.2009.405
43. Nøjgaard C, Becker U, Matzen P, Andersen JR, Holst C, Bendtsen F. Progression from acute to chronic pancreatitis: prognostic factors, mortality, and natural course. Pancreas. 2011;40(8):1195-200. https://doi.org/10.1097/MPA.0b013e318221f569

44. Yadav D, O’Connell M, Papachristou GI. Natural history following the first attack of acute pancreatitis. Am J Gastroenterol. 2012;107(7):1096-103. https://doi.org/10.1038/ajg.2012.126

45. Seidensticker F, Otto J, Lankisch PG. Recovery of the pancreas after acute pancreatitis is not necessarily complete. Int J Pancreatol. 1995;17(3):225-9. https://doi.org/10.1007/BF02785818 